\title{
RECOMBINATION PARAMETERS OF SOME FABRICATED IONIZATION CHAMBERS
}

\author{
Afia Begum $^{1}$ and Nobuhisa Takata ${ }^{2}$ \\ ${ }^{1}$ Department of Physics, Bangladeah University of Engineering \& Technology, Dhaka, Bangladesh \\ ${ }^{2}$ Ionizing Radiation Section, NMIJ, AIST, Tsukuba, Ibaraki 305-8568, Japan \\ email: afia2412@yahoo.com
}

\begin{abstract}
Some pancake and spherical type ionization chambers of various size have been designed and fabricated for absolute air kerma measurement in ${ }^{60} \mathrm{Co}$ and ${ }^{137} \mathrm{Cs} \gamma$-ray fields at the Primary Standard Dosimetry Laboratory (PSDL), National Institute of Advanced Industrial Science $\&$ Technology (AIST), Tsukuba, Japan. Values of recombination parameters A and $\mathrm{m}^{2} \mathrm{~g}$ of these ionization chambers are obtained using a method proposed by De Almeida and Niatel and adopted by Boutillon. For absolute air kerma measurement, it is important to obtain accurate signal currents by correcting it for ion losses using the values of $\mathrm{A}$ and $\mathrm{m}^{2} \mathrm{~g}$ of each of the ionization chambers. It has been observed from the measurements that the recombination parameters for pancake ionization chamber are smaller than spherical ionization chambers and for spherical type ionization chamber recombination parameter values depend on the dimensions of the electrodes and also the size of the chambers.
\end{abstract}

Keywords: Recombination parameter, Ionization chamber, air kerma measurement

\section{INTRODUCTION}

Ionization chambers are used in a wide range of air kerma rates. Response of an ionization chamber depends on the chamber design [1]. When absolute air kerma is measured by an ionization chamber, the effect of incomplete charge collection has been considered. The incomplete charge collection corresponds to initial recombination, volume recombination and back diffusion of ions to electrodes. The dominant process of ion loss is volume recombination [2]. Initial recombination occurs when the positive and negative ions formed in the track of a single ionizing particle meet and recombine. Initial recombination is an intratrack process and independent of the number of tracks per second i.e., of the dose rate. Initial recombination depends only on the initial ion density in each track and the field strength normal to the tracks which tends to pull the positive and negative ions column apart. Volume recombination occurs when the positive and negative ions formed by different ionizing particles meet and recombine as they drift towards the oppositely charged electrodes. Thus the amount of volume recombination increases with the dose rate. Diffusion loss is due to the back diffusion of positive and negative ions to anode and cathode respectively.

In the present work, ion losses due to volume recombination, initial recombination and back diffusion for four spherical type and two pancake type ionization chambers are obtained using a method proposed by De Almeida and Niatel [3] and adopted by Boutillon [4]. In this method all the ion losses are obtained simultaneously by measuring the signal currents at different applied voltages in different air kerma rates.

\section{BASIC EQUATIONS}

At near saturation i.e., when the ion losses due to recombination and diffusion are small, the ratio between the saturation current $I_{S}$ and the ionization current $I_{V}$ measured at an applied voltage $V$, can be obtained by the eqn. (neglecting the terms of higher order)

with

$$
I_{S} / I_{V}=1+A / V+m^{2}\left(g / V^{2}\right) I_{S}
$$

$$
m^{2}=\alpha /\left(e k_{+} k_{-}\right)
$$


where $A$ is constant for a particular chamber involved in the initial recombination and back diffusion, $m^{2} g$ is also constant for a particular chamber involved in the volume recombination process, $g$ is a factor which depends on the chamber geometry, $\alpha$ is the volume recombination coefficient under continuous irradiation, $e$ is the charge per ion, $k_{+}$and $k_{-}$are the mobility of the positive and negative ions respectively.

The first variable term on the right-hand side of eqn. (1) describes the loss of ions by initial recombination and diffusion and the second variable term describes the volume recombination loss. So, volume recombination loss depends on applied voltage, air kerma rates and the chamber geometry factor $g$.

If $V$ in eqn. (1) is replaced by the lower voltage $V / n$, a similar expression is obtained for the signal current $I_{V / n}$. Here $n$ is an arbitrary value larger than 1 .

Now dividing this eqn. by the eqn. (1) we may get (neglecting the higher order terms)

$$
I_{V} / I_{V / n}=1+(n-1) A / V+\left(n^{2}-1\right) m^{2}\left(g / V^{2}\right) I_{V}
$$

Currents $I_{V}$ and $I_{V / n}$ are measured at several different air kerma rates and then the values of $I_{V} / I_{V / n}$ are plotted as a function of $I_{V}$. The applied voltage must be high enough to ensure high ion collection efficiency over the whole range of measured currents.

For an ideal parallel plate ionization chamber the factor $\mathrm{g}$ is given by $g=d^{4} / 6$, where $d$ is the separation between the parallel electrodes. And for an ideal spherical ionization chamber in which both the outer electrode and the central electrode are spherical shape of radii $a, b$ respectively

$$
g=\frac{\left[(a-b) \kappa_{s p h}\right]^{4}}{6}
$$

with

$$
\kappa_{s p h}=\left[\frac{1}{3}(a / b+1+b / a)\right]^{1 / 2}
$$

However, in the present case of the fabricated ionization chambers, the chambers are not ideal one and thus it is not possible to obtain the value of $g$ from the above expressions.

\section{IONIZATON CHAMBERS AND MEASUREMENTS}

The spherical and pancake ionization chambers were made of Poco graphite (density $1.82 \mathrm{~g} \mathrm{~cm}^{-3}$ ) and was constructed at the Primary Standard Dosimetry Laboratory (PSDL), AIST, Tsukuba, Japan. In order to measure the ion loss within each of the ionization chambers signal currents are measured in ${ }^{60} \mathrm{Co} \gamma$-ray field for S7A, S60A, S900A, P9A and P60A ionization chambers and for S60B in ${ }^{137} \mathrm{Cs} \gamma$-ray field. The walls of chamber S60B is thick enough for electronic equilibrium in ${ }^{137} \mathrm{Cs} \gamma$-ray and not in ${ }^{60} \mathrm{Co} \gamma$-rays.

The applied voltages $V$ and $V / n$ are 1000, 500, 200,100V for S7A; 1000, 600, 300, 200, 100V for S60A and S60B; 1000, 600, 400, 300V for S900A; 400, 200, 100, 50V for P9A and 700, 300, 100, 30, $10 \mathrm{~V}$ for P60A ionization chambers. Air kerma rates are varied by placing $\mathrm{Cu}$ plates with the total thickness ranging from 6 to $60 \mathrm{~mm}$ or by tungsten (W) disk of $25 \mathrm{~mm}$ thickness at the collimator of the 
${ }^{60} \mathrm{Co}$ source or by an iron disk placed at the collimator of the ${ }^{137} \mathrm{Cs} \gamma$-ray source. The measured signal current is corrected for the temperature $295.15 \mathrm{~K}$ and pressure $101.325 \mathrm{kPa}$, but no correction is made for the dependence of the signal current on humidity. Air humidity ranged from $38 \%$ to $44 \%$ during the measurement of ionization current from all the fabricated ionization chambers at different applied voltages. Signal current is measured at least three times for a particular applied voltage in each polarity. Therefore, for a particular value of applied voltage there are six values of ionization currents at both polarities. Average of the absolute value of the signal currents measured at both polarities of the applied voltage is used as the signal current in order to obtain the effect of ion losses within the chamber.

\section{RESULTS AND DISCUSSIONS}

Fig. 1 shows variation of the signal current ratio $I_{V} / I_{V / n}$ with $I_{V}$ obtained for the ionization chamber S60A. The value of $A$ is obtained from the intercept on the y-axis by the lines fitted to the plotted data and the volume recombination factor $m^{2} g$ is obtained from the slope of the lines. Table 1 shows the values of $A$ and $m^{2} g$ for the S60A ionization chamber at different applied voltages. It is observed from the results that the values of $A$ and $m^{2} g$ for a particular ionization chamber are scattered in a relatively wide range. One of the reasons is that the values of $A$ and $m^{2} g$ is not constant for a particular chamber. Thus change depending on the lifetime of ions [5], i.e. and also depend on the applied voltage of the ionization chamber. Another important reason for such scattering is that these values have been obtained from very small differences of signal currents measured at different applied voltages and different air kerma rates.

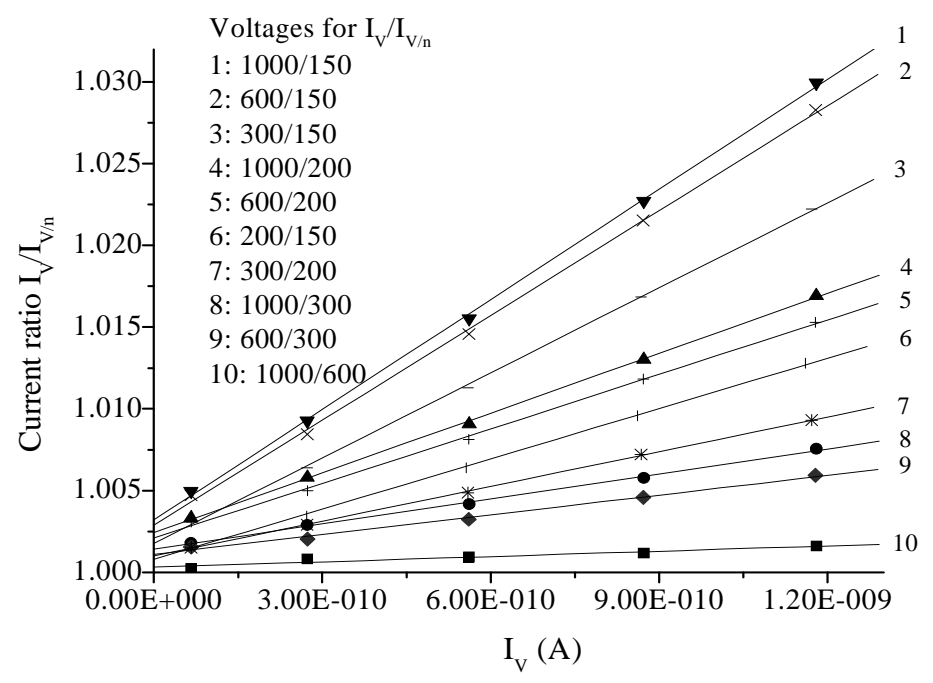

Fig. 1: Ratios of the signal currents measured at different applied voltages for the S60A Ionization Chamber are shown as a function of the current measured for higher applied voltages

In order to observe the dependence of ion loss with the chamber size of spherical chamber, a spherical ionization chamber of volume $V_{s}$ with the spherical wall outer radius $a$ and collector radius $b$ as shown in Fig. 2 is considered.

If $V$ is the applied voltage to the chamber then at a distance $r$ from the center of the chamber the electric field strength $E_{r}$ is 


$$
E_{r}=\frac{V}{r^{2}(1 / b-1 / a)}
$$

Table 1: Values of $A$ and $m^{2} g$ for S60A ionization chamber

\begin{tabular}{|c|c|c|c|}
\hline $\begin{array}{c}V \\
\text { (Volt) }\end{array}$ & $\begin{array}{c}V / n \\
(\text { Volt })\end{array}$ & $A(\mathrm{~V})$ & $m^{2} g\left(\mathrm{~s} \mathrm{C}^{-1} \mathrm{~V}^{2}\right)$ \\
\hline & 600 & $0.48 \pm 0.187$ & $(6.057 \pm 0.985) \mathrm{E}+11$ \\
& 300 & $0.609 \pm 0.039$ & $(5.042 \pm 0.126) \mathrm{E}+11$ \\
1000 & 200 & $0.605 \pm 0.029$ & $(5.082 \pm 0.068) \mathrm{E}+11$ \\
& 100 & $0.566 \pm 0.037$ & $(5.171 \pm 0.068) \mathrm{E}+11$ \\
\hline \multirow{3}{*}{600} & 300 & $0.66 \pm 0.078$ & $(4.825 \pm 0.218) \mathrm{E}+11$ \\
& 200 & $0.63 \pm 0.051$ & $(5.002 \pm 0.107) \mathrm{E}+11$ \\
& 100 & $0.578 \pm 0.057$ & $(5.132 \pm 0.096) \mathrm{E}+11$ \\
\hline \multirow{2}{*}{300} & 200 & $0.594 \pm 0.033$ & $(5.111 \pm 0.056) \mathrm{E}+11$ \\
& 100 & $0.534 \pm 0.046$ & $(5.207 \pm 0.065) \mathrm{E}+11$ \\
\hline 200 & 100 & $0.474 \pm 0.070$ & $(5.271 \pm 0.086) \mathrm{E}+11$ \\
\hline
\end{tabular}

Table 2: Average values of $A$ and $m^{2} g$ for various ionization chambers

\begin{tabular}{|l|l|l|l|}
\hline $\begin{array}{c}\text { Ionization chamber } \\
\text { type (model) }\end{array}$ & $\begin{array}{c}\text { Cavity volume } \\
\left(\mathrm{cm}^{3}\right)\end{array}$ & $A(\mathrm{~V})$ & $\mathrm{m}^{2} g\left(\mathrm{~s} \mathrm{C}^{-1} \mathrm{~V}^{2}\right)$ \\
\hline Spherical S7A & 7.24 & $0.526 \pm 0.018$ & $(2.658 \pm 0.025) \mathrm{E}+11$ \\
\hline Spherical S60A & 65.10 & $0.587 \pm 0.028$ & $(5.136 \pm 0.053) \mathrm{E}+11$ \\
\hline Spherical S60B & 65.10 & $0.655 \pm 0.016$ & $(5.355 \pm 0.147) \mathrm{E}+11$ \\
\hline Spherical S900A & 899.68 & $1.259 \pm 0.087$ & $(1.158 \pm 0.091) \mathrm{E}+12$ \\
\hline Pancake P9A & 8.90 & $0.259 \pm 0.004$ & $(7.759 \pm 2.130) \mathrm{E}+8$ \\
\hline Pancake P60A & 60.70 & $0.107 \pm 0.001$ & $(1.838 \pm 0.082) \mathrm{E}+8$ \\
\hline
\end{tabular}

The average electric field strength $E_{a v a}$ within the chamber volume is considered as the electric field strength varies with the distance $r$ from the center of the spherical chamber.

The average field strength $E_{\text {ava }}$ within the spherical volume of the chamber is

$$
\begin{aligned}
& E_{a v a}=\int_{b}^{a} \frac{4 \pi r^{2} V}{r^{2}(1 / b-1 / a)} d r /\left(\frac{4 \pi}{3}\left(a^{3}-b^{3}\right)\right) \\
& =\frac{4 \pi V a b}{(a-b)} \int_{b}^{a} d r /\left(\frac{4 \pi}{3}\left(a^{3}-b^{3}\right)\right)=\frac{3 a b V}{\left(a^{3}-b^{3}\right)}
\end{aligned}
$$

If $b<<a$, then neglecting $b^{3}$ in the denominator

$$
E_{a v a}=\frac{3 b V}{a^{2}}
$$

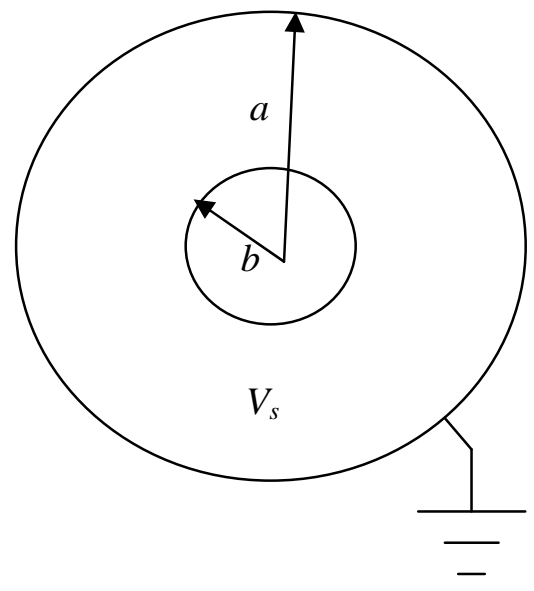

Figure 2: Cross-sectional view of the spherical ionization chamber 
As the initial recombination loss is inversely proportional to the electric field strength and volume recombination loss is inversely proportional to the square of the electric field strength, both initial and volume recombination loss depend on the ratio of the outer and inner electrode radii and also the size of the spherical ionization chamber. Again since average electric field strength within the spherical ionization chamber becomes smaller for larger size ionization chambers, the initial and volume recombination loss become larger for larger size spherical ionization chambers.

Ions are lost by back diffusion if they are produced near the vicinity of the electrode where the electrostatic potential difference from the electrode is less than $k T / e$, where $e$ is the elementary charge, $k$ the Boltzmann constant and $T$ the absolute temperature of air within the chamber. Since the electric field strength near the central electrode is high, as a result the diffusion loss is negligible there, we can neglect the diffusion loss at the central electrode. So, the back diffusion loss takes place mainly at the surface of the outer electrode of the chamber.

The electric field strength at the outer electrode of the spherical chamber $E_{a}$ is given by

$$
E_{a}=\frac{V}{a^{2}(1 / b-1 / a)}=\frac{a b V}{a^{2}(a-b)}=\frac{b V}{a(a-b)}
$$

Since for spherical ionization chamber, diffusion loss is inversely proportional to the electric field strength at the outer electrode $E_{a}$ and the surface area of the outer electrode. The ion loss due to diffusion compared to the total ion-pairs produced within the ionization volume is proportional to

$$
\frac{a(a-b)}{b V} 4 \pi a^{2} /\left(\frac{4 \pi}{3}\left(a^{3}-b^{3}\right)\right)=\frac{3 a^{3}(a-b)}{b V\left(a^{3}-b^{3}\right)}=\frac{3 a^{3}}{b V\left(a^{2}+a b+b^{2}\right)}
$$

If $b<<a$ then it becomes $\frac{3 a}{b V}$

Therefore, ion loss due to diffusion compared to the total ion pairs produced in the ionization volume of the chamber also depends on the ratio between the outer and inner electrode radii of the spherical ionization chamber.

The average values of $A$ and $m^{2} g$ for different ionization chambers are shown in Table 2 are obtained from the values of $A$ and $m^{2} g$ at different applied voltages and $n$ values by weighing with the inverse of uncertainties of the results. It can be observed from the table that the values of $A$ and $m^{2} g$ are larger for larger volume spherical ionization chambers, viz, in the order of S7A, S60A(B), and S900A. The reason for the increase of these values is expected due to the differences in the ratio between the outer electrode radius and the collector electrode radius (which are: $12 / 1=12$ for $\mathrm{S} 7 \mathrm{~A}, 25 / 2.5=10$ for $\mathrm{S} 60 \mathrm{~A}$ (B), and $60 / 6=10$ for $\mathrm{S} 900 \mathrm{~A}$ ); and the outer electrode radius. If the applied voltages to all these spherical chambers are same then the diffusion loss in S7A is greater than that of other spherical chambers such as S60A, S60B and S900A and the ion loss due to diffusion is same for S60A, S60B and S900A ionization chambers.

Since for larger volume spherical chamber the initial recombination loss is larger, so if the applied voltage to all these chambers S60A, S60B and S900A are same then the initial recombination loss in S900A ionization chamber is larger than $\mathrm{S} 60 \mathrm{~A}(\mathrm{~B})$ ionization chambers. For this reason it has been seen from Table 2 that the value of $A$ for S900A is larger than that of the other spherical chambers S60A and S60B. 
Separation distance between the electrodes of pancake ionization chambers P9A and P60A are very small which are $(4.8-0.3) / 2=2.25 \mathrm{~mm}$ and $(8-0.3) / 2=3.85 \mathrm{~mm}$ respectively. In the case of P9A Ionization Chamber, the uncertainty in the measurement of recombination parameter is large. This is due to very small loss of signal currents from the chamber. In the case of pancake ionization chambers, both the values for $A$ and $m^{2} g$ are larger for the smaller chamber P9A than for the larger chamber P60A. This may be due to large ion losses at the corners of the ionization volume where the electric field strength is relatively small. The percentage of the corner volume is larger for the smaller ionization chamber, P9A, than that for the larger chamber P60A.

\section{CONCLUSIONS}

From the results it is observed that for spherical type ionization chambers recombination parameters depend on the dimensions of the electrodes and also the size of the chambers. The values of recombination parameters $A$ and $m^{2} g$ for pancake chambers are larger for smaller chamber and it is smaller than that for spherical chambers.

\section{REFERENCES}

1. Attix FH: Introduction to Radiological Physics and Radiation Dosimetry. John Wiley \& Sons, New York, 1986.

2. Boag JW: Ionization Measurements at very high intensities. br. J. Radiol. 23: 601, 1950.

3. De Almeida CE, Niatel MT: Comparison between IRD and BIPM exposure and air kerma standards for cobalt gamma rays. BIPM Report 12: 1, 1986.

4. Boutillon M: Volume recombination parameter in ionization chambers. Phys. Med. Biol. 43: 2061, 1998.

5. Takata $\mathrm{N}$ and Matiullah: Dependence of the value of $m$ on the lifetime of ions in parallel-plate ionization chambers. Phys. Med. Biol. 36: 449, 1991 